\title{
The prognostic value of SUMO1/Sentrin specific peptidase 1 (SENP1) in prostate cancer is limited to ERG-fusion positive tumors lacking PTEN deletion
}

Christoph Burdelski ${ }^{1+}$, Devi Menan ${ }^{2 \dagger}$, Maria Christina Tsourlakis ${ }^{2}$, Martina Kluth², Claudia Hube-Magg ${ }^{2}$, Nathaniel Melling ${ }^{1}$, Sarah Minner ${ }^{2}$, Christina Koop ${ }^{2}$, Markus Graefen ${ }^{3}$, Hans Heinzer $^{3}$, Corinna Wittmer ${ }^{2}$, Guido Sauter ${ }^{2}$, Ronald Simon ${ }^{2}$, Thorsten Schlomm ${ }^{3,4}$, Stefan Steurer ${ }^{2}$ and Till Krech ${ }^{2 *}$

\begin{abstract}
Background: Posttranscriptional protein modification by SUMOylation plays an important role in tumor development and progression. In the current study we analyzed prevalence and prognostic impact of the de-SUMOylation enzyme SENP1 in prostate cancer.

Methods: SENP1 expression was analyzed by immunohistochemistry on a tissue microarray containing more than 12,400 prostate cancer specimens. Results were compared to tumor phenotype, ERG status, genomic deletions of 3p, $5 \mathrm{q}, 6 \mathrm{q}$ and PTEN, and biochemical recurrence.

Results: SENP1 immunostaining was detectable in $34.5 \%$ of 9,516 interpretable cancers and considered strong in $7.3 \%$, moderate in $14.9 \%$ and weak in $12.3 \%$ of cases. Strong SENP1 expression was linked to advanced pT stage $(p<0.0001)$, high Gleason grade $(p<0.0001)$, positive lymph node status $(p=0.0019)$, high pre-operative PSA levels $(p=0.0037)$, and PSA recurrence $(p<0.0001)$. SENP1 expression was strongly associated with positive ERG fusion status as determined by both in situ hybridization (FISH) and immunohistochemistry as well as with PTEN deletions. Detectable SENP1 immunostaining was found in $41 \%$ of ERG positive and in $47 \%$ of PTEN deleted cancers but in only $30 \%$ of ERG negative and $30 \%$ of PTEN non-deleted cancers ( $p<0.0001$ each). Deletions of 3p, 5q, and $6 q$ were unrelated to SENP1 expression. Subset analyses revealed that the prognostic impact of SENP1 expression was solely driven by the subgroup of ERG positive, PTEN undeleted cancers. In this subgroup, the prognostic role of SENP1 expression was independent of the preoperative PSA level, tumor stage, Gleason grade, and the status of the resection margin.
\end{abstract}

Conclusions: SENP1 expression has strong prognostic impact in a molecularly defined subset of cancers. This is per se not surprising as the biologic impact of each individual molecular event is likely to be dependent on its cellular environment. However, such findings challenge the concept of finding clinically relevant molecular signatures that are equally applicable to all prostate cancers.

Keywords: Prostate cancer, ERG fusion, PTEN deletion, SENP1, SUMO, Immunohistochemistry, Tissue microarray

\footnotetext{
*Correspondence: t.krech@uke.de

${ }^{\dagger}$ Equal contributors

${ }^{2}$ Institute of Pathology, University Medical Center Hamburg-Eppendorf,

Hamburg, Germany

Full list of author information is available at the end of the article
}

\section{Biomed Central}

(c) 2015 Burdelski et al. This is an Open Access article distributed under the terms of the Creative Commons Attribution License (http://creativecommons.org/licenses/by/4.0), which permits unrestricted use, distribution, and reproduction in any medium, provided the original work is properly credited. The Creative Commons Public Domain Dedication waiver (http:// creativecommons.org/publicdomain/zero/1.0/) applies to the data made available in this article, unless otherwise stated. 


\section{Background}

Prostate cancer is the most prevalent cancer in men in Western societies [1]. Although most prostate cancers have a rather indolent clinical course, this disease still represents the third most common cause of cancer related death in men. A reliable distinction between the indolent and the aggressive forms of the disease is highly desirable to enhance therapeutic decisions. Despite recent advances, the only established pretreatment prognostic parameters currently include Gleason grade and tumor extent on biopsies, preoperative prostate-specific antigen (PSA), and clinical stage. Because these data are statistically powerful but not sufficient for optimal individual treatment decisions, it can be hoped that a better understanding of disease biology will eventually lead to the identification of clinically applicable molecular markers that enable a more reliable prediction of prostate cancer aggressiveness.

SUMOylation is a revertible posttranscriptional protein modification involving the binding of small ubiquitin-like modifiers (SUMOs) to target proteins. SUMOs are structurally related to ubiquitin and are covalently attached to target proteins by a SUMOconjugating system resembling the ubiquitination machinery [2]. SUMOylation affects protein stability and activity, and regulates a variety of cellular processes, such as nuclear transport, transcription, and apoptosis [3]. Several proteins control the balance between SUMOylation and de-SUMOylation. A key protein for de-SUMOylation is SUMO1/Sentrin specific peptidase 1 (SENP1) [4], which deconjugates SUMOs from a large number of SUMOylated proteins [5]. Since important target genes of SENP1 include histone deacetylases and cell cycle regulators like cyclin D1, SENP1 is also involved in control of epigenetic transcription and cell proliferation [6-10]. Consequently, overexpression of SENP1 has been found in various cancer types [10], such as colon cancer [11], bladder cancer [12], head \& neck cancer [13], and lung cancer [14], and has been linked to poor clinical features in some of these $[13,15]$. In the prostate gland, SENP1 was shown to act as a transcriptional activator of androgen receptor (AR) signaling [7]. Two studies analyzing SENP1 in 115 and 150 Asian prostate cancer patients suggested that SENP1 overexpression might be an independent marker of poor prognosis $[16,17]$.

These promising findings prompted us to study the putative prognostic value of SENP1 expression measurement in a large cohort including more than 12,400 European prostate cancers that have been assembled in a tissue microarray (TMA) format. The database attached to our TMA contains pathological and clinical follow-up data, as well molecular data of key molecular alterations of this disease such as ERG fusion and genomic deletion of PTEN, 3p13, 5q21, and 6q15, which were used to establish associations between SENP1 expression and distinct phenotypic and molecular subsets of prostate cancers.

\section{Methods \\ Patients}

Radical prostatectomy specimens were available from 12,427 patients, undergoing surgery between 1992 and 2012 at the Department of Urology and the Martini Clinics at the University Medical Center HamburgEppendorf. Follow-up data were available for a total of 11,665 patients with a median follow-up of 36 months (range: 1 to 241 months; Table 1). Prostate specific antigen (PSA) values were measured following surgery and PSA recurrence was defined as a postoperative PSA of $\geq$ $0.2 \mathrm{ng} / \mathrm{ml}$ confirmed by a second determination with a serum PSA $\geq 0.2 \mathrm{ng} / \mathrm{ml}$. All prostate specimens were analyzed according to a standard procedure, including a complete embedding of the entire prostate for histological analysis [18].

The TMA manufacturing process was described earlier in detail [19]. In short, one $0.6 \mathrm{~mm}$ core was taken from a representative tissue block from each patient. The tissues were distributed among 27 TMA blocks, each containing 144 to 522 tumor samples. For internal controls, each TMA block also contained various control tissues, including normal prostate tissue. The molecular database attached to this TMA contained results on ERG expression in 10,711 [20], ERG break apart FISH analysis in 7,122 (expanded from [21]) and deletion status of 5q21 (CHD1) in 7932 (expanded from [22]), 6q15 (MAP3K7) in 6,069 (expanded from [23]), 10q23 (PTEN) in 6,704 (expanded from [24]) and 3p13 (FOXP1) in 7,081 (expanded from [25]) cancers. Immunohistochemical data on Ki67 labeling index (LI) were available from 7,010 cancers (expanded from [26]).

The usage of archived diagnostic left-over tissues for manufacturing of tissue microarrays and their analysis for research purposes as well as patient data analysis has been approved by the local ethics committee (Ethics commission Hamburg, WF-049/09 and PV3652). All work has been carried out in compliance with the Helsinki Declaration.

Usage of patient data and routinely archived formalin fixed left-over patient tissue samples for research purposes by the attending physician is approved by local laws and does not require written consent (HmbKHG, $\$ 12,1$ ).

\section{Immunohistochemistry}

Freshly cut TMA sections were immunostained on one day and in one experiment. Slides were deparaffinized and exposed to heat-induced antigen retrieval for $5 \mathrm{~min}$ in an autoclave at $121{ }^{\circ} \mathrm{C}$ in $\mathrm{pH} 7.8$ Tris-EDTA-Citrate buffer. 
Table 1 Pathological and clinical data of the arrayed prostate cancers. Percentage in the column "Study cohort on TMA" refers to the fraction of samples across each category. Percentage in column "Biochemical relapse among categories" refers to the fraction of samples with biochemical relapse within each parameter in the different categories. Numbers do not always add up to 12,427 in the different categories because of cases with missing data. Abbreviation: AJCC, American Joint Committee on Cancer

\begin{tabular}{|c|c|c|}
\hline & \multicolumn{2}{|l|}{ No. of patients (\%) } \\
\hline & $\begin{array}{l}\text { Study cohort on } \\
\text { TMA }(n=12427)\end{array}$ & $\begin{array}{l}\text { Biochemical relapse } \\
\text { among categories }\end{array}$ \\
\hline \multicolumn{3}{|c|}{ Follow-up (mo) } \\
\hline$n$ & 11665 (93.9 \%) & 2769 (23.7 \%) \\
\hline Mean & 48.9 & - \\
\hline Median & 36.4 & - \\
\hline \multicolumn{3}{|l|}{ Age (y) } \\
\hline$\leq 50$ & $334(2.7 \%)$ & 81 (24.3\%) \\
\hline $51-59$ & 3061 (24.8 \%) & 705 (23\%) \\
\hline $60-69$ & 7188 (58.2 \%) & 1610 (22.4 \%) \\
\hline$\geq 70$ & 1761 (14.3\%) & $370(21 \%)$ \\
\hline \multicolumn{3}{|c|}{ Pretreatment PSA (ng/ml) } \\
\hline$<4$ & 1585 (12.9 \%) & $242(15.3 \%)$ \\
\hline $4-10$ & 7480 (60.9 \%) & 1355 (18.1%) \\
\hline $10-20$ & 2412 (19.6 \%) & 737 (30.6 \%) \\
\hline$>20$ & $812(6.6 \%)$ & 397 (48.9 \%) \\
\hline \multicolumn{3}{|c|}{ pT category (AJCC 2002) } \\
\hline pT2 & 8187 (66.2 \%) & 1095 (13.4 \%) \\
\hline рТ3а & 2660 (21.5 \%) & 817 (30.7 \%) \\
\hline pT3b & 1465 (11.8 \%) & $796(54.3 \%)$ \\
\hline pT4 & $63(0.5 \%)$ & $51(81 \%)$ \\
\hline \multicolumn{3}{|c|}{ Gleason grade } \\
\hline$\leq 3+3$ & $2983(24.1 \%)$ & $368(12.3 \%)$ \\
\hline $3+4$ & 6945 (56.2 \%) & 1289 (18.6 \%) \\
\hline $4+3$ & 1848 (15 \%) & $788(42.6 \%)$ \\
\hline$\geq 4+4$ & $584(4.7 \%)$ & 311 (53.3 \%) \\
\hline \multicolumn{3}{|c|}{ pN category } \\
\hline pNO & 6970 (91 \%) & 1636 (23.5 \%) \\
\hline $\mathrm{pN}+$ & $693(9 \%)$ & $393(56.7 \%)$ \\
\hline \multicolumn{3}{|c|}{ Surgical margin } \\
\hline Negative & 9990 (81.9 \%) & 1848 (18.5 \%) \\
\hline Positive & $2211(18.1 \%)$ & $853(38.6 \%)$ \\
\hline
\end{tabular}

Primary antibody specific for SENP1 (rabbit monoclonal antibody, EPR3844, Abcam, Cambridge, UK; cat\#108981; dilution 1:150) was applied at $37{ }^{\circ} \mathrm{C}$ for $60 \mathrm{~min}$. Bound antibody was then visualized using the EnVision Kit (Dako, Glostrup, Denmark) according to the manufacturer's directions. Staining was predominantly nuclear and typically accompanied by cytoplasmic co-staining. The intensity of the cytoplasmic staining was usually weaker than the intensity of nuclear staining. Nuclear and cytoplasmic SENP1 staining was typically found in either all $(100 \%)$ or none $(0 \%)$ of the tumor cells in a given cancer spot. Staining intensity of all cases was thus semiquantitatively assessed in four categories: negative, weak, moderate and strong. The percentage of positive tumor cells (typically $100 \%$ ) was not separately recorded. An additional isotype control (rabbit monoclonal, SP137, Abcam, Cambridge, UK; cat\#128142) yielded no unspecific staining (data not shown).

\section{Statistics}

For statistical analysis, the JMP ${ }^{\circledR}$ 10.0.2 software (2012 SAS Institute Inc., NC, USA) was used. Contingency 
tables were calculated to study association between SENP1 staining and clinico-pathological variables, and the Chi-squared (Likelihood) test was used to find significant relationships. Kaplan Meier curves were generated for PSA recurrence free survival. The log-Rank test was applied to test the significance of differences between stratified survival functions. Cox proportional hazards regression analysis was performed to test the statistical independence and significance between pathological, molecular, and clinical variables.

\section{Results}

\section{Technical issues}

A total of 9,516 (77 \%) of tumor samples were interpretable in our TMA analysis. Reason for non-informative cases (2,911 spots; $23 \%)$ included lack of tissue samples or absence of unequivocal cancer tissue in the TMA spot.

\section{SENP1 immunohistochemistry}

In normal prostatic glands, weak cytoplasmic staining was found in almost all cases, whereas nuclear staining was rare and occurred in only two out of 20 (10\%) cases. Positive staining was limited to the secretory epithelial cells, while basal cells were consistently negative. In cancers, SENP1 immunostaining was predominantly localized in the nucleus. Positive staining was seen in 3,283 of our 9,516 (34.5\%) interpretable tumors and was considered weak in $12.3 \%$, moderate in $14.9 \%$ and strong in $7.3 \%$ of cancers. Representative images of positive and negative SENP1 immunostainings are given in Fig. 1. Strong SENP1 immunostaining was significantly linked to advanced pathological tumor stage $(\mathrm{p}<0.0001)$, high Gleason grade $(\mathrm{p}<0.0001)$, presence of lymph node metastases $(p=0.0019)$ and high preoperative PSA-levels $(p=0.0037)$ when all tumors were jointly analyzed (Table 2). SENP1 immunostaining showed no significant association with positive resection margin status $(p=0.3216)$.

\section{Association with TMPRSS2:ERG fusion status and ERG protein expression}

To evaluate whether SENP1 expression is associated with ERG status in prostate cancers, we used data from previous studies (expanded from [20, 21]). Data on
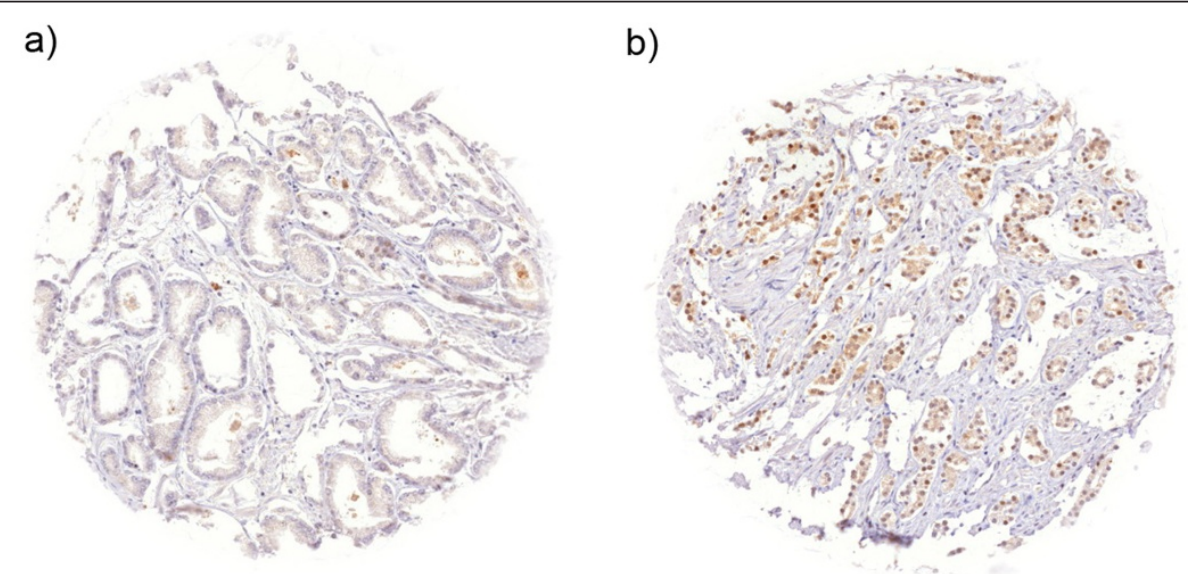

c)

d)
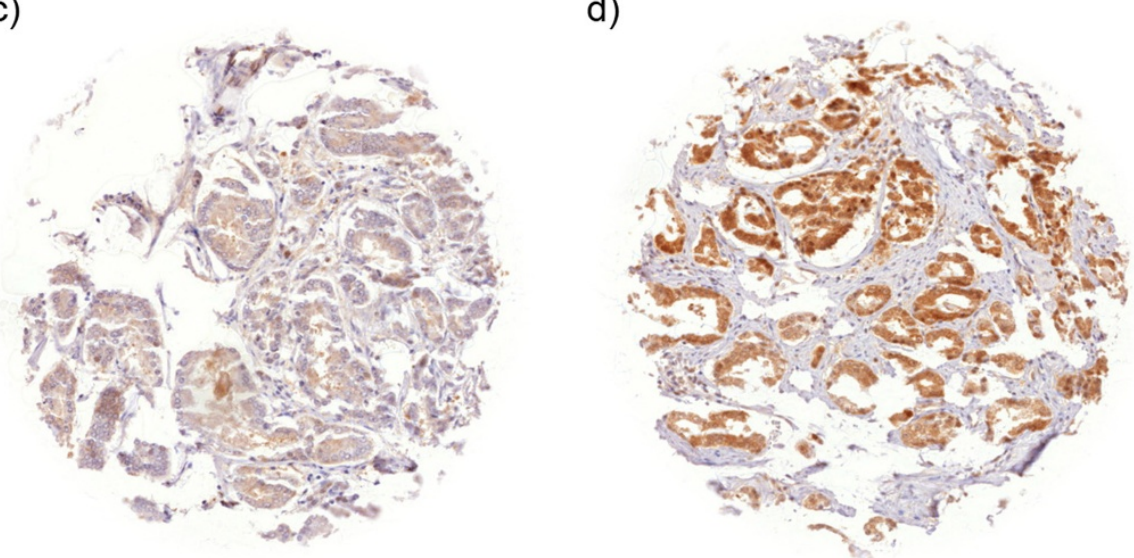

Fig. 1 Representative pictures of SENP1 immunostaining in prostate cancer with a) negative, b) weak, c) moderate, and d) strong staining 
Table 2 Association between SENP1 immunostaining results and prostate cancer phenotype in all cancers

\begin{tabular}{|c|c|c|c|c|c|c|}
\hline \multirow[t]{2}{*}{ Parameter } & \multicolumn{5}{|l|}{ SENP1 } & \multirow[t]{2}{*}{$\mathrm{p}$ value } \\
\hline & n evaluable & $\begin{array}{l}\text { Negative } \\
(\%)\end{array}$ & $\begin{array}{l}\text { Weak } \\
(\%)\end{array}$ & $\begin{array}{l}\text { Moderate } \\
(\%)\end{array}$ & $\begin{array}{l}\text { Strong } \\
(\%)\end{array}$ & \\
\hline All cancers & 9,516 & 65.5 & 12.3 & 14.9 & 7.3 & \\
\hline Tumor stage & & & & & & $<0.0001$ \\
\hline pT2 & 6,143 & 68.2 & 11.1 & 14.1 & 6.6 & \\
\hline рT3a & 2,137 & 61.8 & 13.7 & 15.9 & 8.7 & \\
\hline pT3b-4 & 1,203 & 58.1 & 15.8 & 17.6 & 8.5 & \\
\hline Gleason grade & & & & & & $<0.0001$ \\
\hline$\leq 3+3$ & 2,135 & 72.5 & 9.2 & 11.6 & 6.7 & \\
\hline $3+4$ & 5,451 & 65.3 & 11.7 & 15.5 & 7.5 & \\
\hline $4+3$ & 1,445 & 58.4 & 16.2 & 17.6 & 7.8 & \\
\hline$\geq 4+4$ & 442 & 58.1 & 20.8 & 14.9 & 6.1 & \\
\hline Lymph node metastasis & & & & & & 0.0019 \\
\hline No & 5,472 & 62.3 & 12.6 & 16.6 & 8.5 & \\
\hline $\mathrm{N}+$ & 526 & 56.8 & 18.4 & 17.5 & 7.2 & \\
\hline Preop. PSA level (ng/ml) & & & & & & 0.0037 \\
\hline$<4$ & 1160 & 64.2 & 12.2 & 15.9 & 7.6 & \\
\hline $4-10$ & 5702 & 66.8 & 11.2 & 15.0 & 7.0 & \\
\hline $10-20$ & 1892 & 63.4 & 14.6 & 14.5 & 7.5 & \\
\hline$>20$ & 666 & 62.3 & 14.9 & 14.3 & 8.6 & \\
\hline Surgical margin & & & & & & 0.3216 \\
\hline negative & 7,549 & 65.9 & 12.1 & 14.9 & 7.1 & \\
\hline positive & 1,797 & 63.8 & 13.0 & 15.2 & 8.0 & \\
\hline
\end{tabular}

TMPRSS2:ERG fusion status obtained by FISH were available from 5,677 and by immunohistochemistry from 8,459 tumors with evaluable SENP1 immunostaining. Data on both ERG FISH and IHC were available from 5,468 cancers, and an identical result (ERG IHC positive and break by FISH or ERG IHC negative and missing break by FISH) was found in 5,231 of 5,468 (95.7 \%) cancers. SENP1 immunostaining was slightly more frequent in TMPRSS2:ERG rearranged and ERG positive prostate cancers than in ERG negative tumors. Positive SENP1 immunostaining was seen in $41.7 \%$ (ERG IHC) and $40.9 \%$ (ERG FISH) of ERG positive cancers but in only $28.6 \%$ and $30 \%$ of cancers without ERG staining and ERG rearrangement, respectively ( $\mathrm{p}<0.0001$ each; Fig. 2). SENP1 immunostaining was similarly linked to unfavorable tumor features in subsets of both ERG negative and ERG positive cancers (Additional file 1: Table S1 and Additional file 2: Table S2).

\section{Association to other key genomic deletions}

Earlier studies had provided evidence for recurrent chromosomal deletions delineating further molecular subgroups amongst ERG positive and ERG negative prostate cancers. In particular, deletions of PTEN and 3p13 define subgroups in ERG positive and deletions of 5 q21 and 6q15 define subgroups in ERG negative cancers $[22,23,25]$. To examine, whether SENP1 expression might be particularly associated with one of these genomic deletions, SENP1 data were compared to preexisting findings on PTEN (10q23), 3p13 (FOXP1), 6q15 (MAP3K7) and 5q21 (CHD1) deletions. Elevated SENP1 expression levels were strongly linked to deletions of PTEN both in ERG positive and ERG negative cancers $(\mathrm{p}<0.0001$ each, Fig. 3). However, SENP1 was largely unrelated to all other deletions irrespective of whether all cancers or subgroups of ERG positive or ERG negative cancers were analyzed.

\section{Association to tumor cell proliferation (Ki67LI)}

Strong SENP1 staining was significantly linked to accelerated cell proliferation as measured by Ki67LI in all cancers $(\mathrm{p}<0.0001)$. This association held also true with high significance in most subgroups of cancers with identical Gleason grade $(\leq 3+3 ; 3+4 ; 4+3 ; \geq 4+4)$, and also in the subset of ERG positive tumors lacking PTEN deletions $(p=0.0315)$. All comparisons with the Ki67LI are summarized in Table 3. 


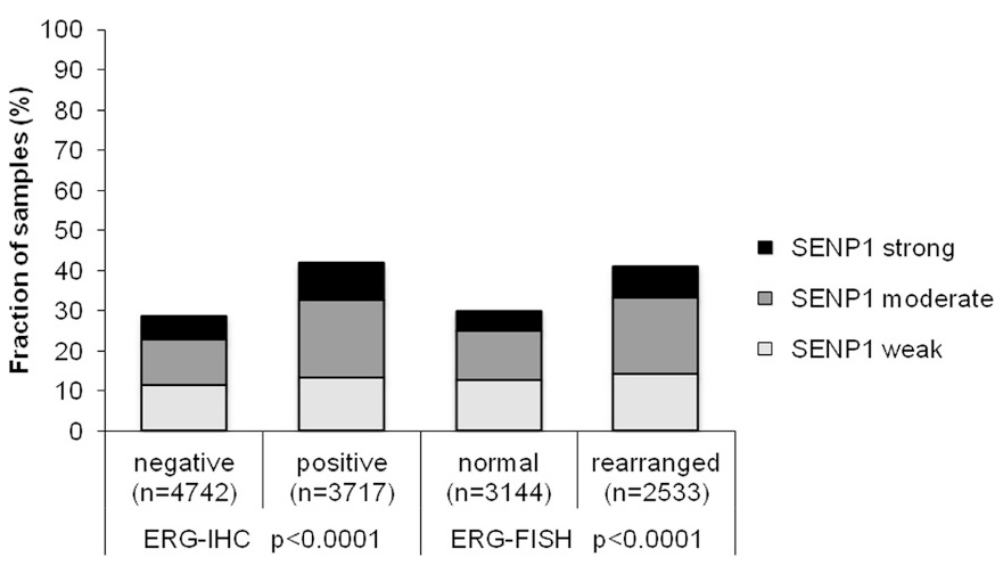

Fig. 2 Association between SENP1 immunostaining results and the ERG-status determined by IHC and FISH analysis. Rearranged indicates breakage of the ERG gene according to FISH analysis

\section{Association with PSA recurrence}

Follow-up data were available from 8,920 patients with interpretable SENP1 immunostaining on the TMA. Since there was no significant prognostic impact of the level of positive SENP1 staining (data not shown), all cancers with weak, moderate, and strong SENP1 staining were combined into one group ("positive") for follow-up analysis. Tumors with positive SENP1 immunostaining showed a significantly shortened PSA recurrence-free interval if all cancers were jointly analyzed ( $\mathrm{p}<0.0001$, Fig. 4a), as well as in subsets of ERG-IHC-positive ( $p<0.0001$, Fig. $4 \mathrm{~b})$ or ERG-IHC-negative cancers $\mathrm{p}<0.0001$, Fig. 4c). Because of the strong link between SENP1 expression and PTEN deletion, we extended the analyses to tumor subgroups stratified according to the SENP1/ PTEN status. These analyses revealed that the prognostic impact of SENP1 expression was limited to cancers lacking PTEN deletions in ERG positive ( $\mathrm{p}<0.0001 \mathrm{Fig} .4 \mathrm{~d})$, but not in ERG negative tumors $(p=0.1251$, Fig. 4e). SENP1 had no prognostic relevance in cancers harboring PTEN deletions, neither in ERG positive ( $p=0.7745$, Fig. $4 \mathrm{~d})$, nor in ERG negative cancers $(p=0.7267$, Fig. $4 \mathrm{e})$.

\section{Multivariate analysis}

Four different types of multivariate analyses were performed evaluating the clinical relevance of SENP1 expression in different scenarios (Table 4). Scenario 1 evaluated all postoperatively available parameters including pathological tumor stage, pathological lymph node status $(\mathrm{pN})$, surgical margin status, preoperative PSA value and pathological Gleason grade obtained after the morphological evaluation of the entire resected prostate. In scenario 2 , all postoperatively available parameters with exception of nodal status were included. The rational for this approach was that the indication and extent of lymph node dissection is not standardized in the surgical therapy of prostate cancer and that excluding $\mathrm{pN}$ in multivariate analysis can markedly increase case numbers. Two additional scenarios had the purpose to model the preoperative situation as much as possible. Scenario 3 included SENP1 expression, preoperative PSA, clinical tumor stage (cT stage) and Gleason grade obtained on the prostatectomy specimen. Since postoperative determination of a tumors Gleason grade is "better" than the preoperatively determined Gleason grade (subjected to sampling errors and consequently under-grading in more than one third of cases [27]), another multivariate analysis was added. In scenario 4, the preoperative Gleason grade obtained on the original biopsy was combined with preoperative PSA, cT stage and SENP1 expression. SENP1 largely did not provide independent prognostic information if all tumors or the subgroups of ERG positive and ERG negative cancers were interrogated. A further subset analysis of ERG positive/ PTEN undeleted cancers revealed independent prognostic impact, however, in 3 of 4 tested scenarios (Table 4 a-d).

\section{Discussion}

Immunohistochemically detectable SENP1 expression was found in about $35 \%$ of prostate cancers in our study. This frequency is lower than what has been observed in two earlier IHC studies, reporting positive SENP1 staining in $76.5 \%$ of 115 [16] and high SENP1 expression in $47 \%$ of 117 [17] analyzed prostate cancers from Asian patients. These earlier studies also analyzed tissue microarrays. Although both previous studies utilized a slightly larger core diameter $(1 \mathrm{~mm})$ than in our study $(0.6 \mathrm{~mm})$, it seems unlikely that the lower fraction of SENP1 positive cancers in our study was caused by sampling bias due to this small difference in core diameter. Rather, different antibodies, immunohistochemistry protocols, and scoring criteria might have contributed to the slightly variable results between these studies. Given the paramount impact of IHC protocols on the positivity rates in TMA studies [18] we would not view our data 

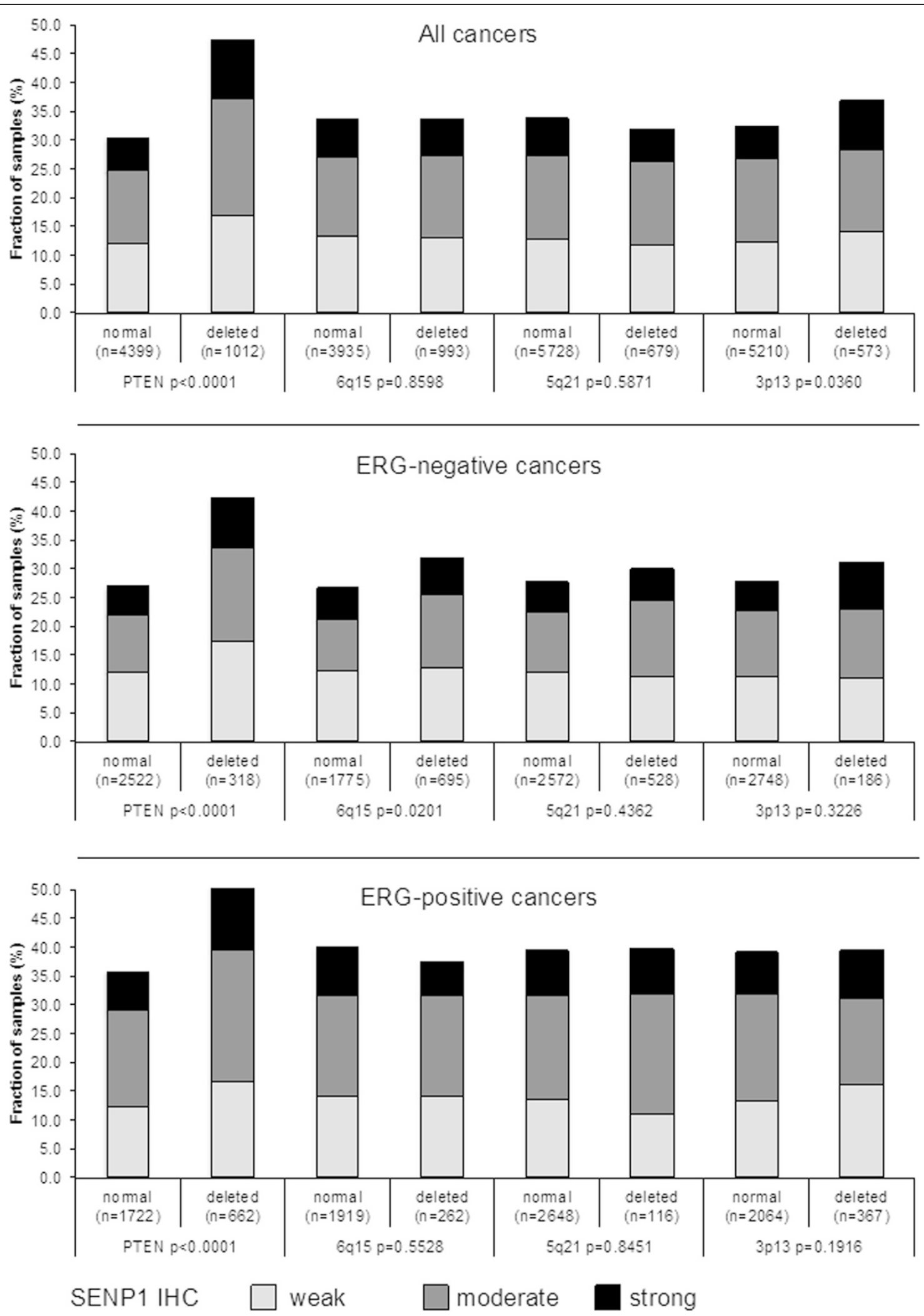

Fig. 3 Association between positive SENP1 immunostaining results and deletions of PTEN, $5 q 21$ (CHD1), 6q15 (MAP3K7), and 3p13 (FOXP1) in all cancers as well as the subsets of ERG-negative and ERG-positive cancers according to ERG-IHC analysis 
Table 3 Associations between SENP1 immunohistochemistry results and cell proliferation as measured by Ki67 immunohistochemistry in all cancers and subsets of cancers defined by Gleason grade, and the ERG/PTEN status. Ki67Llav = average Ki67 labeling index. * P-value for SENP1 negative vs. positive (combined groups of weak, moderate, strong)

\begin{tabular}{|c|c|c|c|c|c|c|}
\hline \multirow{2}{*}{ All cancers } & & \multirow{2}{*}{$\begin{array}{l}\text { SENP1 IHC } \\
\text { negative }\end{array}$} & \multirow{2}{*}{$\begin{array}{l}\text { Number } \\
3,880\end{array}$} & \multicolumn{2}{|c|}{ Ki67LI av } & \multirow[t]{2}{*}{$P$} \\
\hline & & & & 2.58 & \pm 0.04 & \\
\hline & & weak & 679 & 3.05 & \pm 0.10 & $<0.0001$ \\
\hline & & moderate & 838 & 3.31 & \pm 0.09 & ${ }^{*}<0.0001$ \\
\hline & & strong & 419 & 3.21 & \pm 0.13 & \\
\hline \multirow[t]{16}{*}{ Gleason } & $\leq 3+3$ & negative & 980 & 2.07 & \pm 0.07 & \\
\hline & & weak & 112 & 2.30 & \pm 0.19 & $<0.0001$ \\
\hline & & moderate & 137 & 2.55 & \pm 0.18 & ${ }^{*}<0.0001$ \\
\hline & & strong & 75 & 2.65 & \pm 0.24 & \\
\hline & $3+4$ & negative & 2,238 & 2.51 & \pm 0.05 & \\
\hline & & weak & 396 & 3.02 & \pm 0.12 & $<0.0001$ \\
\hline & & moderate & 520 & 3.14 & \pm 0.10 & ${ }^{*}<0.0001$ \\
\hline & & strong & 252 & 3.24 & \pm 0.15 & \\
\hline & $4+3$ & negative & 504 & 3.34 & \pm 0.16 & \\
\hline & & weak & 119 & 3.69 & \pm 0.32 & 0.4329 \\
\hline & & moderate & 137 & 3.85 & \pm 0.30 & ${ }^{*} 0.1209$ \\
\hline & & strong & 71 & 3.56 & \pm 0.42 & \\
\hline & $\geq 4+4$ & negative & 133 & 4.74 & \pm 0.39 & \\
\hline & & weak & 51 & 3.41 & \pm 0.63 & 0.0516 \\
\hline & & moderate & 41 & 5.90 & \pm 0.70 & ${ }^{*} 0.5643$ \\
\hline & & strong & 18 & 3.78 & \pm 1.06 & \\
\hline \multirow[t]{4}{*}{ ERG-positive cancers without PTEN deletion } & & negative & 814 & 2.92 & \pm 0.09 & \\
\hline & & weak & 151 & 3.44 & \pm 0.21 & 0.0315 \\
\hline & & moderate & 196 & 2.99 & \pm 0.19 & *0.0293 \\
\hline & & strong & 80 & 3.59 & \pm 0.29 & \\
\hline
\end{tabular}

as strong evidence in favor of possible ethnical differences in SENP1 expression in prostate cancers.

Our analysis revealed weak cytoplasmic SENP1 staining in secretory cells of normal prostate epithelium, while more intense cytoplasmic and nuclear staining was rare and occurred in only about $10 \%$ of normal tissues. Finding a markedly higher fraction of cytoplasmic/nuclear SENP1 staining in cancer as compared to normal prostate suggests that SENP1 becomes upregulated in a fraction of tumors. Comparable to our observation, $\mathrm{Li}$ et al. [16] reported a gradual increase of SENP1 positivity from normal prostate $(4.2 \%)$ to prostatic intraepithelial neoplasia (PIN, $57.9 \%$ ) and cancer (76.5\%). SENP1 expression was significantly linked to adverse tumor features including advanced stage, high Gleason grade, and presence of lymph node metastases, preoperative PSA levels, and early biochemical recurrence in our analysis. These findings are in line with earlier studies in prostate cancer reporting significant associations with advanced and high-grade cancers as well as poor prognosis in Asian patients $[16,17]$. Similar results have also been observed in analyses of other solid cancer types, including cancers of the colon [11], bladder [12], head \& neck [13], and lung [14], where SENP1 overexpression was consistently linked to advanced and high-grade cancers and in some studies also with adverse clinical outcome $[11,13]$. A relevant tumor biological role of SENP1 is also supported by our observation that SENP1 expression was linked to increased cell proliferation. Known biological functions of SENP1 are consistent with a role in cancer development and progression. SENP1 activity affects the homeostasis of post-transcriptional SUMO modification of various target proteins required for normal cell physiology. While both loss of SUMO conjugation as well as excessive SUMOylation results in embryonic lethality $[28,29]$, more subtle changes of the SUMOylation machinery lead to deregulation of multiple cellular pathways including those with relevance for cell proliferation and differentiation [10]. Genes and pathways known to be targeted by SENP1 include histone deacetylases [7], c-Jun- and ERK-dependent transcription [30, 31], cyclin D1 activity [32], Pi3K/AKT 
b)

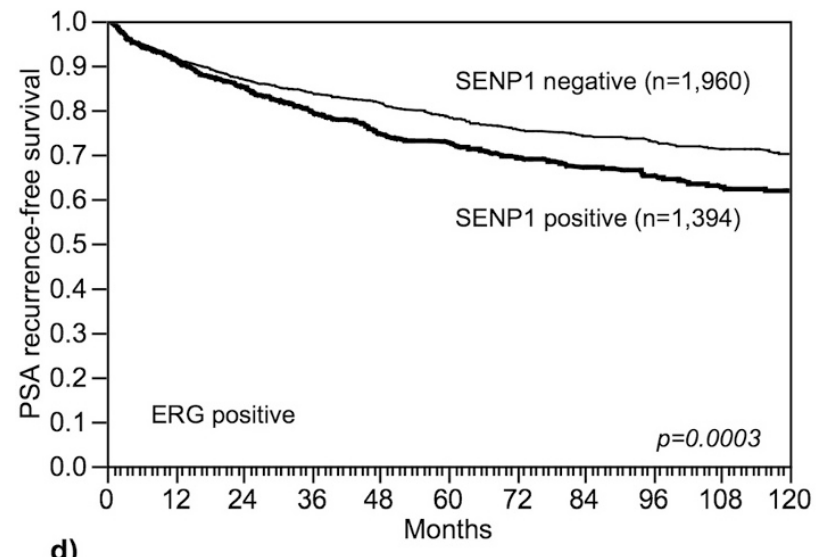

d)

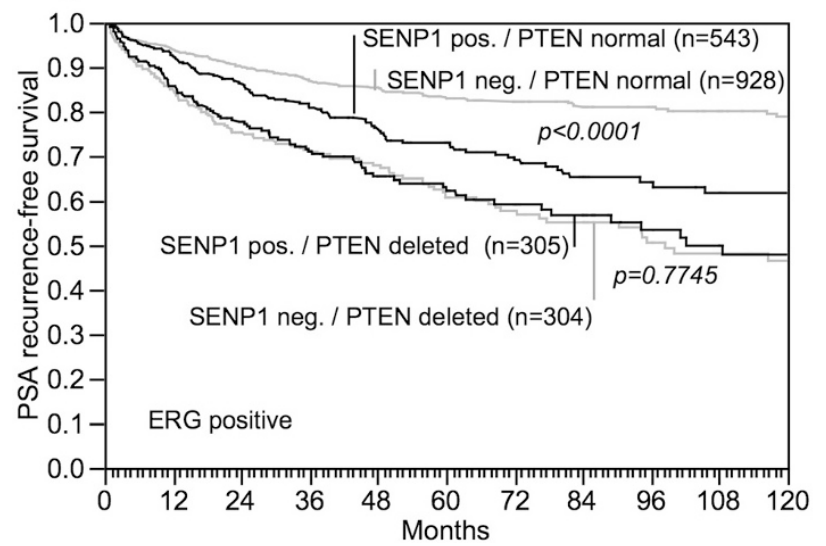

a)

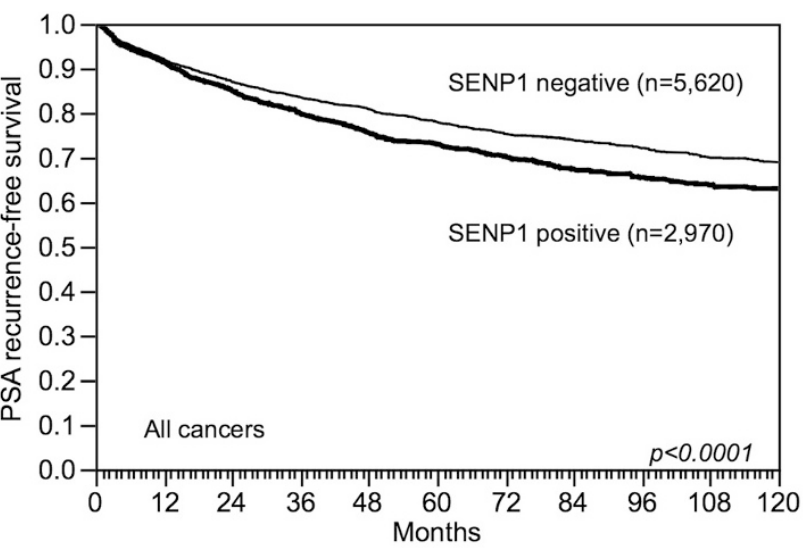

c)

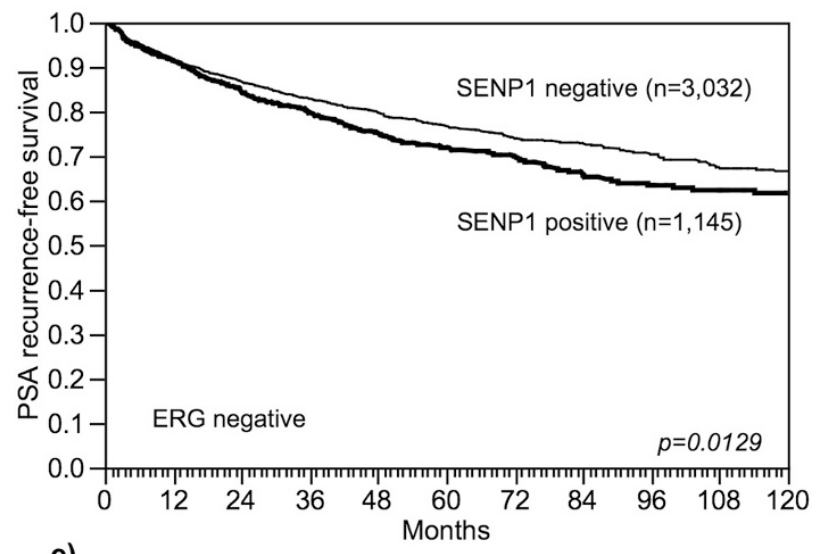

e)

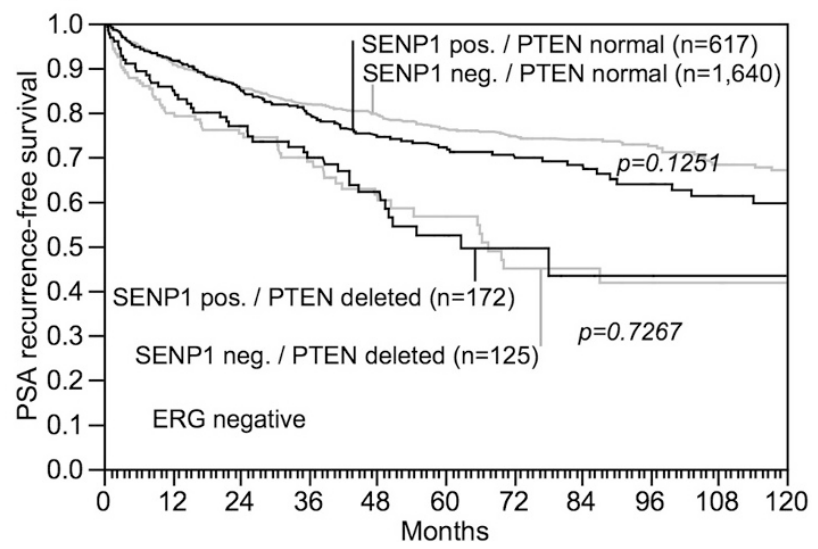

Fig. 4 Association between SENP1 expression and biochemical recurrence in a) all cancers, b) ERG-HHC positive cancers, $\mathbf{c}$ ) ERG-IHC negative cancers. Combined effect of SENP1 and PTEN deletion in $\mathbf{d}$ ) all cancers, e) ERG-IHC positive cancers and $\mathbf{f}$ ) ERG-IHC negative cancers

signaling pathway $[33,34]$, and HIF $1 \alpha$-dependent angiogenesis [29].

The high number of tumors in our TMA enabled us to profoundly evaluate SENP1 in the context of key genomic alterations of prostate cancer. Gene fusions involving the androgen-regulated serine protease TMPRSS2 and ERG, a member of the ETS family of transcription factors, occur in about $50 \%$ of prostate cancers and result in strong AR-driven ERG protein overexpression $[35,36]$ and massive transcriptional changes [37-40]. The increased SENP1 expression levels in ERG positive cancers detected by two independent approaches (i.e. ERG-IHC and -FISH) in our study apparently reflects the AR dependency of both SENP1 and ERG, since 
Table 4 Multivariate analysis including SENP1 expression in a) all cancers, b) ERG-negative, c) ERG-positive cancers and d) ERG-positive cancers lacking PTEN deletion

\begin{tabular}{|c|c|c|c|c|c|c|c|c|c|}
\hline \multicolumn{10}{|l|}{ a) } \\
\hline \multirow[t]{2}{*}{ Scenario } & \multirow[t]{2}{*}{ n analyzable } & \multicolumn{8}{|l|}{$p$-value } \\
\hline & & $\begin{array}{l}\text { Preoperative } \\
\text { PSA-Level }\end{array}$ & $\begin{array}{l}\text { pT } \\
\text { Stage }\end{array}$ & $\begin{array}{l}\mathrm{CT} \\
\text { Stage }\end{array}$ & $\begin{array}{l}\text { Gleason grade } \\
\text { prostatectomy }\end{array}$ & $\begin{array}{l}\text { Gleason grade } \\
\text { biopsy }\end{array}$ & N-Stage & R-Status & $\begin{array}{l}\text { SENP1 } \\
\text { Expression }\end{array}$ \\
\hline 1 & 5,273 & $<0.0001$ & $<0.0001$ & - & $<0.0001$ & - & 0.0001 & 0.0008 & 0.9255 \\
\hline 2 & 8,392 & $<0.0001$ & $<0.0001$ & - & $<0.0001$ & - & - & $<0.0001$ & 0.9136 \\
\hline 3 & 8,268 & $<0.0001$ & - & $<0.0001$ & $<0.0001$ & - & - & - & 0.6842 \\
\hline 4 & 8,155 & $<0.0001$ & - & $<0.0001$ & - & $<0.0001$ & - & - & 0.0227 \\
\hline \multicolumn{10}{|l|}{ b) } \\
\hline \multirow[t]{2}{*}{ Scenario } & $\mathrm{n}$ analyzable & \multicolumn{8}{|l|}{$p$-value } \\
\hline & & $\begin{array}{l}\text { Preoperative } \\
\text { PSA-Level }\end{array}$ & pT Stage & CT Stage & $\begin{array}{l}\text { Gleason-grade } \\
\text { prostatectomy }\end{array}$ & $\begin{array}{l}\text { Gleason grade } \\
\text { biopsy }\end{array}$ & $\mathrm{N}$-Stage & R-Status & $\begin{array}{l}\text { SENP1 } \\
\text { Expression }\end{array}$ \\
\hline 1 & 2,681 & 0.0004 & $<0.0001$ & - & $<0.0001$ & - & 0.0006 & 0.1375 & 0.1487 \\
\hline 2 & 4,179 & $<0.0001$ & $<0.0001$ & - & $<0.0001$ & - & - & 0.0022 & 0.1962 \\
\hline 3 & 4,145 & $<0.0001$ & - & $<0.0001$ & $<0.0001$ & - & - & - & 0.3180 \\
\hline 4 & 4,091 & $<0.0001$ & - & $<0.0001$ & - & $<0.0001$ & - & - & 0.3539 \\
\hline \multicolumn{10}{|l|}{ c) } \\
\hline \multirow[t]{2}{*}{ Scenario } & $\mathrm{n}$ analyzable & \multicolumn{8}{|l|}{$p$-value } \\
\hline & & $\begin{array}{l}\text { preoperative } \\
\text { PSA-Level }\end{array}$ & pT Stage & CT Stage & $\begin{array}{l}\text { Gleason-grade } \\
\text { prostatectomy }\end{array}$ & $\begin{array}{l}\text { Gleason grade } \\
\text { biopsy }\end{array}$ & N-Stage & R-Status & $\begin{array}{l}\text { SENP1 } \\
\text { Expression }\end{array}$ \\
\hline 1 & 2,090 & 0.0003 & $<0.0001$ & - & $<0.0001$ & - & 0.0073 & 0.0065 & 0.4108 \\
\hline 2 & 3,279 & $<0.0001$ & $<0.0001$ & - & $<0.0001$ & - & - & $<0.0001$ & 0.4351 \\
\hline 3 & 3,203 & $<0.0001$ & - & $<0.0001$ & $<0.0001$ & - & - & - & 0.3231 \\
\hline 4 & 3,156 & $<0.0001$ & - & $<0.0001$ & - & $<0.0001$ & - & - & 0.0143 \\
\hline \multicolumn{10}{|l|}{ d) } \\
\hline \multirow[t]{2}{*}{ Scenario } & n analyzable & \multicolumn{8}{|l|}{$p$-value } \\
\hline & & $\begin{array}{l}\text { preoperative } \\
\text { PSA-Level } \\
\end{array}$ & pT Stage & cT Stage & $\begin{array}{l}\text { Gleason-grade } \\
\text { prostatectomy }\end{array}$ & $\begin{array}{l}\text { Gleason grade } \\
\text { biopsy }\end{array}$ & N-Stage & R-Status & $\begin{array}{l}\text { SENP1 } \\
\text { Expression } \\
\end{array}$ \\
\hline 1 & 872 & 0.0015 & $<0.0001$ & - & $<0.0001$ & - & 0.0007 & 0.0958 & 0.1174 \\
\hline 2 & 1,495 & 0.0012 & $<0.0001$ & - & $<0.0001$ & - & - & 0.0017 & 0.0157 \\
\hline 3 & 1,463 & $<0.0001$ & - & 0.0197 & $<0.0001$ & - & - & - & 0.0057 \\
\hline 4 & 1,444 & $<0.0001$ & - & 0.0354 & - & $<0.0001$ & - & - & 0.0005 \\
\hline
\end{tabular}

SENP1 functions both as a transcriptional target as well as an inducer of AR expression in a positive feedback loop $[32,41]$.

Further subgroup analyses targeted highly recurrent chromosomal deletions that are tightly linked to the ERG status and that may delineate important molecular subgroups within ERG positive and ERG negative cancers. For example, 3p13 and PTEN deletions are linked to ERG positivity and deletions at $5 \mathrm{q} 21$ and $6 \mathrm{q} 15$ to ERG negativity and all these deletions have high prognostic impact within these subgroups [23-25, 42-44]. This analysis revealed that SENP1 expression was not only linked to a positive ERG status but to an even stronger extent to PTEN deletions. The classical function of PTEN involves control of the PI3K/AKT signaling pathway by antagonizing PI3K activity [45]. A functional relationship of PTEN and SENP1 is conceivable because SENP1 induced SUMOylation is known to occur and to have biological impact in the PTEN/PI3K/ AKT signaling pathway [33, 34]. Comparison of large enough molecularly defined subgroups with clinical data is one approach to further interrogate functional interrelationships "in vivo". The complete lack of a difference in clinical outcome between PTEN deleted cancers with and without SENP1 expression argues against a clinically relevant cooperative effect of reduced PTEN function and SENP1 activation. The very strong association between SENP1 overexpression and PTEN would, however, be consistent with models suggesting a role of SENP1 activation for development of PTEN deletions. This could be driven by the effect of SENP1 on histone modification and its impact on the epigenetic machinery. 
Both histone configuration and epigenetic events are thought to predispose to the development of specific genomic alterations including deletions [46-48]. In such a scenario, the additional PTEN deletion would result in such a strong disruption of cancer cell physiology that SENP1 expression no longer has a critical additional effect on tumor aggressiveness.

The overall prognostic impact of SENP1 expression was - although statistically highly significant - rather small in absolute numbers. Several models for multivariate analyses were used in this study in order to - as much as possible - model the application of prognostic features in pre- and postoperative scenarios. Unfortunately, in the real world, prognostic molecular features can hardly be analyzed on preoperative biopsies because these are typically distributed among many different pathology laboratories, and even if they were available for analyses such precious collection of tissues would be used up after only a few studies. The application of multivariate models revealed that SENP1 largely lacked independent prognostic value if all tumors and the classical molecular subgroups of ERG positive and ERG negative cancers were analyzed. However, our subgroup analyses demonstrated that the significant impact of SENP1 expression on outcome was entirely driven by the subgroup of ERG positive PTEN non-deleted cancers. Accordingly, independent prognostic relevance was seen for SENP1 expression in this particular subgroup. In earlier studies, we have identified other molecular markers that seemed to exert their prognostic impact only in specific molecularly defined subgroups such as in ERG positive and PTEN deleted cancers $[49,50]$, ERG negative cancers lacking PTEN deletion [51], ERG positive cancers [25], ERG negative cancers [52], cancers lacking PTEN deletion $[53,54]$, or in all cancers irrespective of ERG and PTEN status [55].

The frequent finding of subtype specific prognostic features challenges the concept of molecular classifiers that apply to all prostate cancers. For example, several multiparametric prognostic tests were recently suggested in prostate cancer [56-59] and several tests are now commercially available to patients $[60,61]$. It might be interesting to see, how these tests perform in molecularly defined prostate cancer subgroups.

With SENP1 being one of the most important deSUMOylating enzymes, it has been hypothesized that targeting SENP1 with inhibitory drugs may restore the balance of the SUMO modification system [10], and several experimental SENP1 specific inhibitors have been successfully designed as to yet $[8,62-64]$. Such inhibitors may even cooperate with other treatment modalities that are commonly used in prostate cancer. Recently, Wang et al. used RNAi for depletion of SENP1 in lung cancer cell lines and found that inhibition of SENP1 markedly enhanced the radiosensitivity of lung carcinoma by promoting irradiation-induced cell cycle arrest, $\gamma$-H2AX expression and apoptosis [14]. Although clinical studies are so far lacking, these first attempts emphasize the potential druggability of SENP1 in human cancers. Given that prostate cancer is characterized by AR-driven SENP1 expression, it is possible that drugs targeting SENP1, possibly in combination with anti-androgenic therapy, will also be effective in prostate cancer.

\section{Conclusions}

Overall, our study demonstrates that SENP1 overexpression is frequent in ERG positive prostate cancer and linked to PTEN deletions. Moreover, SENP1 overexpression has strong prognostic value in the subset of ERGpositive prostate cancers lacking PTEN deletions.

\section{Additional files}

Additional file 1: Table S1. Association between SENP1

immunostaining results and prostate cancer phenotype in ERG-fusion negative tumors. (DOC $63 \mathrm{~kb}$ )

Additional file 2: Table S2. Association between SENP1 immunostaining results and prostate cancer phenotype in ERG-fusion positive tumors. (DOC $63 \mathrm{~kb}$ )

\section{Abbreviations}

SUMO: Small ubiquitin-like modifiers; SENP1: SUMO1/Sentrin specific peptidase 1; ERG: v-ets avian erythroblastosis virus E26 oncogene related; PSA: Prostate-specific antigen; FISH: Fluorescence In Situ Hybridization; PTEN: Phosphatase and tensin homolog; TMA: Tissue micro array; CHD1: Chromodomain helicase DNA binding protein 1; MAP3K7: Mitogen-activated protein kinase kinase kinase 7; FOXP1: Forkhead box P1; Ki67: Marker of proliferation Ki-67; IHC: Immunohistochemistry; PI3K: Phosphatidylinositol-4,5-bisphosphate 3-kinase; AKT: v-akt murine thymoma viral oncogene homolog 1; HIF1a: Hypoxia-inducible factor 1-alpha; ETS family: (erythroblast transformation- specific) family of transcription factors; AR: Androgen receptor; RNAi: RNA interference.

\section{Competing interests}

The authors declare that they have no competing interests.

\section{Authors' contributions}

CB, DM, C H-M, GS, RS and TK conceived and designed the study, analyzed the data and drafted the manuscript. CB and DM performed most of the key immunohistochemical analyses. GS and RS were involved in the original conception of the study. MCT, MK, NM, C H-M and SM provided data. CB, $\mathrm{DM}, \mathrm{MCT}, \mathrm{SM}, \mathrm{CK}, \mathrm{CW}, \mathrm{GS}, \mathrm{SS}$ and TK participated in tissue processing, pathological diagnosis and immunohistochemical analysis. CK, MG, HH, GS, RS and TS provided materials, clinical follow-up data and technical assistance. All authors have read and approved the manuscript.

\section{Acknowledgements}

We thank Sophia Krech and Stefan Kraft for their support, discussion, and thoughtful feedback. This work was supported by the Institute of Pathology, University Medical Center Hamburg-Eppendorf, Germany.

\section{Author details}

${ }^{1}$ General, Visceral and Thoracic Surgery Department and Clinic, University Medical Center Hamburg-Eppendorf, Hamburg, Germany. ${ }^{2}$ Institute of Pathology, University Medical Center Hamburg-Eppendorf, Hamburg, Germany. ${ }^{3}$ Martini-Clinic, Prostate Cancer Center, University Medical Center Hamburg- Eppendorf, Martinistr. 25, 20246 Hamburg, Germany. ${ }^{4}$ Department 
of Urology, Section for translational Prostate Cancer Research, University Medical Center Hamburg-Eppendorf, Hamburg, Germany.

Received: 26 November 2014 Accepted: 14 July 2015 Published online: 23 July 2015

\section{References}

1. Siegel R, Naishadham D, Jemal A. Cancer statistics, 2013. CA Cancer J Clin. 2013;63(1):11-30.

2. Hay RT. SUMO: a history of modification. Mol Cell. 2005;18(1):1-12.

3. Geiss-Friedlander R, Melchior F. Concepts in sumoylation: a decade on. Nat Rev Mol Cell Biol. 2007;8(12):947-56.

4. Guo D, Li M, Zhang Y, Yang P, Eckenrode S, Hopkins D, et al. A functional variant of SUMO4, a new I kappa B alpha modifier, is associated with type 1 diabetes. Nat Genet. 2004;36(8):837-41.

5. Gong L, Millas S, Maul GG, Yeh ET. Differential regulation of sentrinized proteins by a novel sentrin-specific protease. J Biol Chem. 2000;275(5):3355-9.

6. Bailey D, O'Hare P. Characterization of the localization and proteolytic activity of the SUMO-specific protease, SENP1. J Biol Chem. 2004;279(1):692-703.

7. Cheng J, Wang D, Wang Z, Yeh ET. SENP1 enhances androgen receptor-dependent transcription through desumoylation of histone deacetylase 1. Mol Cell Biol. 2004;24(13):6021-8.

8. Huang W, He T, Chai C, Yang Y, Zheng Y, Zhou P, et al. Triptolide inhibits the proliferation of prostate cancer cells and down-regulates SUMO-specific protease 1 expression. PLoS One. 2012;7(5):e37693.

9. Kaikkonen S, Jaaskelainen T, Karvonen U, Rytinki MM, Makkonen H, Gioeli D, et al. SUMO-specific protease 1 (SENP1) reverses the hormone-augmented SUMOylation of androgen receptor and modulates gene responses in prostate cancer cells. Mol Endocrinol. 2009;23(3):292-307.

10. Bawa-Khalfe T, Yeh ET. SUMO Losing Balance: SUMO Proteases Disrupt SUMO Homeostasis to Facilitate Cancer Development and Progression. Genes Cancer. 2010;1(7):748-52.

11. Xu Y, Li J, Zuo Y, Deng J, Wang LS, Chen GQ. SUMO-specific protease 1 regulates the in vitro and in vivo growth of colon cancer cells with the upregulated expression of CDK inhibitors. Cancer Lett. 2011;309(1):78-84.

12. Brems-Eskildsen AS, Zieger K, Toldbod H, Holcomb C, Higuchi R, Mansilla F, et al. Prediction and diagnosis of bladder cancer recurrence based on urinary content of hTERT, SENP1, PPP1CA, and MCM5 transcripts. BMC Cancer. 2010;10:646.

13. Ni RS, Shen X, Qian X, Yu C, Wu H, Gao X. Detection of differentially expressed genes and association with clinicopathological features in laryngeal squamous cell carcinoma. Oncol Lett. 2012;4(6):1354-60.

14. Wang RT, Zhi XY, Zhang Y, Zhang J. Inhibition of SENP1 induces radiosensitization in lung cancer cells. Exp Ther Med. 2013;6(4):1054-8.

15. Park HC, Seong J, An JH, Kim J, Kim UJ, Lee BW. Alteration of cancer pain-related signals by radiation: proteomic analysis in an animal model with cancer bone invasion. Int J Radiat Oncol Biol Phys. 2005;61 (5):1523-34.

16. Ponce DM, Sauter C, Devlin S, Lubin M, Gonzales AM, Kernan NA, et al. A Novel Reduced-Intensity Conditioning Regimen Induces a High Incidence of Sustained Donor-Derived Neutrophil and Platelet Engraftment after DoubleUnit Cord Blood Transplantation. Biol Blood Marrow Transplant. 2013.

17. Wang Q, Xia N, Li T, Xu Y, Zou Y, Zuo Y, et al. SUMO-specific protease 1 promotes prostate cancer progression and metastasis. Oncogene. 2013;32(19):2493-8.

18. Schlomm T, Iwers L, Kirstein P, Jessen B, Kollermann J, Minner S, et al. Clinical significance of p53 alterations in surgically treated prostate cancers. Mod Pathol. 2008;21(11):1371-8.

19. Kononen J, Bubendorf L, Kallioniemi A, Barlund M, Schraml P, Leighton S, et al. Tissue microarrays for high-throughput molecular profiling of tumor specimens. Nat Med. 1998;4(7):844-7.

20. Weischenfeldt J, Simon R, Feuerbach L, Schlangen K, Weichenhan D, Minner $S$, et al. Integrative genomic analyses reveal an androgen-driven somatic alteration landscape in early-onset prostate cancer. Cancer Cell. 2013;23(2):159-70.

21. Minner S, Enodien M, Sirma H, Luebke AM, Krohn A, Mayer PS, et al. ERG status is unrelated to PSA recurrence in radically operated prostate cancer in the absence of antihormonal therapy. Clin Cancer Res. 2011;17(18):5878-88.
22. Burkhardt L, Fuchs S, Krohn A, Masser S, Mader M, Kluth M, et al. CHD1 is a 5 q21 tumor suppressor required for ERG rearrangement in prostate cancer. Cancer Res. 2013;73(9):2795-805.

23. Kluth M, Hesse J, Heinl A, Krohn A, Steurer S, Sirma H, et al. Genomic deletion of MAP3K7 at 6q12-22 is associated with early PSA recurrence in prostate cancer and absence of TMPRSS2:ERG fusions. Mod Pathol. 2013;26(7):975-83.

24. Krohn A, Diedler T, Burkhardt L, Mayer PS, De Silva C, Meyer-Kornblum M, et al. Genomic deletion of PTEN is associated with tumor progression and early PSA recurrence in ERG fusion-positive and fusion-negative prostate cancer. Am J Pathol. 2012;181(2):401-12.

25. Krohn A, Seidel A, Burkhardt L, Bachmann F, Mader M, Grupp K, et al. Recurrent deletion of $3 p 13$ targets multiple tumour suppressor genes and defines a distinct subgroup of aggressive ERG fusion-positive prostate cancers. J Pathol. 2013;231(1):130-41.

26. Minner S, Jessen B, Stiedenroth L, Burandt E, Kollermann J, Mirlacher M, et al. Low level HER2 overexpression is associated with rapid tumor cell proliferation and poor prognosis in prostate cancer. Clin Cancer Res. 2010;16(5):1553-60.

27. Epstein Jl, Feng Z, Trock BJ, Pierorazio PM. Upgrading and downgrading of prostate cancer from biopsy to radical prostatectomy: incidence and predictive factors using the modified Gleason grading system and factoring in tertiary grades. Eur Urol. 2012;61(5):1019-24.

28. Nacerddine K, Lehembre F, Bhaumik M, Artus J, Cohen-Tannoudji M, Babinet C, et al. The SUMO pathway is essential for nuclear integrity and chromosome segregation in mice. Dev Cell. 2005;9(6):769-79.

29. Cheng J, Kang X, Zhang S, Yeh ET. SUMO-specific protease 1 is essential for stabilization of HIF1alpha during hypoxia. Cell. 2007;131(3):584-95.

30. Cheng J, Perkins ND, Yeh ET. Differential regulation of c-Jun-dependent transcription by SUMO-specific proteases. J Biol Chem. 2005;280(15):14492-8

31. Kubota Y, O'Grady P, Saito H, Takekawa M. Oncogenic Ras abrogates MEK SUMOylation that suppresses the ERK pathway and cell transformation. Nat Cell Biol. 2011;13(3):282-91.

32. Jiang Z, Fan Q, Zhang Z, Zou Y, Cai R, Wang Q, et al. SENP1 deficiency promotes ER stress-induced apoptosis by increasing XBP1 SUMOylation. Cell Cycle. 2012;11(6):1118-22.

33. Huang J, Yan J, Zhang J, Zhu S, Wang Y, Shi T, et al. SUMO1 modification of PTEN regulates tumorigenesis by controlling its association with the plasma membrane. Nat Commun. 2012;3:911.

34. Choe CU, Nabuurs C, Stockebrand MC, Neu A, Nunes P, Morellini F, et al. L-arginine:glycine amidinotransferase deficiency protects from metabolic syndrome. Hum Mol Genet. 2013;22(1):110-23.

35. Tomlins SA, Rhodes DR, Perner S, Dhanasekaran SM, Mehra R, Sun XW, et al. Recurrent fusion of TMPRSS2 and ETS transcription factor genes in prostate cancer. Science. 2005;310(5748):644-8.

36. Tomlins SA, Mehra R, Rhodes DR, Smith LR, Roulston D, Helgeson BE, et al. TMPRSS2:ETV4 gene fusions define a third molecular subtype of prostate cancer. Cancer Res. 2006;66(7):3396-400.

37. Jhavar S, Reid A, Clark J, Kote-Jarai Z, Christmas T, Thompson A, et al. Detection of TMPRSS2-ERG translocations in human prostate cancer by expression profiling using GeneChip Human Exon 1.0 ST arrays. J Mol Diagn. 2008;10(1):50-7.

38. Gupta S, Iljin K, Sara H, Mpindi JP, Mirtti T, Vainio P, et al. FZD4 as a mediator of ERG oncogene-induced WNT signaling and epithelial-to-mesenchymal transition in human prostate cancer cells. Cancer Res. 2010;70(17):6735-45.

39. Brase JC, Johannes M, Mannsperger H, Falth M, Metzger J, Kacprzyk LA, et al. TMPRSS2-ERG -specific transcriptional modulation is associated with prostate cancer biomarkers and TGF-beta signaling. BMC Cancer. 2011;11:507.

40. Ijin K, Wolf M, Edgren H, Gupta S, Kilpinen S, Skotheim Rl, et al. TMPRSS2 fusions with oncogenic ETS factors in prostate cancer involve unbalanced genomic rearrangements and are associated with HDAC1 and epigenetic reprogramming. Cancer Res. 2006;66(21):10242-6.

41. Bawa-Khalfe T, Cheng J, Wang Z, Yeh ET. Induction of the SUMO-specific protease 1 transcription by the androgen receptor in prostate cancer cells. J Biol Chem. 2007;282(52):37341-9.

42. Lapointe J, Li C, Giacomini CP, Salari K, Huang S, Wang P, et al. Genomic profiling reveals alternative genetic pathways of prostate tumorigenesis. Cancer Res. 2007:67(18):8504-10.

43. Taylor BS, Schultz N, Hieronymus H, Gopalan A, Xiao Y, Carver BS, et al. Integrative genomic profiling of human prostate cancer. Cancer Cell. 2010;18(1):11-22. 
44. Berger MF, Lawrence MS, Demichelis F, Drier Y, Cibulskis K, Sivachenko AY, et al. The genomic complexity of primary human prostate cancer. Nature. 2011;470(7333):214-20.

45. Worby CA, Dixon JE. Pten. Annu Rev Biochem. 2014;83:641-69.

46. Dovey OM, Foster CT, Conte N, Edwards SA, Edwards JM, Singh R, et al. Histone deacetylase 1 and 2 are essential for normal T-cell development and genomic stability in mice. Blood. 2013;121(8):1335-44.

47. Li X, Liu L, Yang S, Song N, Zhou X, Gao J, et al. Histone demethylase $\mathrm{KDM5B}$ is a key regulator of genome stability. Proc Natl Acad Sci U S A. 2014:111(19):7096-101.

48. Lahue RS, Frizzell A. Histone deacetylase complexes as caretakers of genome stability. Epigenetics. 2012;7(8):806-10

49. Grupp K, Ospina-Klinck D, Tsourlakis MC, Koop C, Wilczak W, Adam M, et al NY-ESO-1 expression is tightly linked to TMPRSS2-ERG fusion in prostate cancer. Prostate. 2014;74(10):1012-22.

50. Muller J, Ehlers A, Burkhardt L, Sirma H, Steuber T, Graefen M, et al. Loss of pSer2448-mTOR expression is linked to adverse prognosis and tumor progression in ERG-fusion-positive cancers. Int J Cancer. 2013;132(6):1333-40.

51. Grupp K, Boumesli R, Tsourlakis MC, Koop C, Wilczak W, Adam M, et al. The prognostic impact of high Nijmegen breakage syndrome (NBS1) gene expression in ERG-negative prostate cancers lacking PTEN deletion is driven by KPNA2 expression. Int J Cancer. 2014;135(6):1399-407.

52. Stumm L, Burkhardt L, Steurer S, Simon R, Adam M, Becker A, et al. Strong expression of the neuronal transcription factor FOXP2 is linked to an increased risk of early PSA recurrence in ERG fusion-negative cancers. J Clin Pathol. 2013;66(7):563-8.

53. Grupp K, Habermann M, Sirma H, Simon R, Steurer S, Hube-Magg C, et al. High nuclear karyopherin alpha 2 expression is a strong and independent predictor of biochemical recurrence in prostate cancer patients treated by radical prostatectomy. Mod Pathol. 2014;27(1):96-106.

54. Grupp K, Sanader S, Sirma H, Simon R, Koop C, Prien K, et al. High lysophosphatidylcholine acyltransferase 1 expression independently predicts high risk for biochemical recurrence in prostate cancers. Mol Oncol. 2013;7(6):1001-11.

55. Tsourlakis MC, Weigand P, Grupp K, Kluth M, Steurer S, Schlomm T, et al. betalll-tubulin overexpression is an independent predictor of prostate cancer progression tightly linked to ERG fusion status and PTEN deletion. Am J Pathol. 2014;184(3):609-17.

56. Erho N, Crisan A, Vergara IA, Mitra AP, Ghadessi M, Buerki C, et al. Discovery and validation of a prostate cancer genomic classifier that predicts early metastasis following radical prostatectomy. PLoS One. 2013;8(6):e66855.

57. Bismar TA, Alshalalfa M, Petersen LF, Teng LH, Gerke T, Bakkar A, et al. Interrogation of ERG gene rearrangements in prostate cancer identifies a prognostic 10-gene signature with relevant implication to patients' clinical outcome. BJU Int. 2014;113(2):309-19.

58. Rizzi F, Belloni L, Crafa P, Lazzaretti M, Remondini D, Ferretti S, et al. A novel gene signature for molecular diagnosis of human prostate cancer by RT-qPCR. PLoS One. 2008;3(10):e3617.

59. Nakagawa T, Kollmeyer TM, Morlan BW, Anderson SK, Bergstralh EJ, Davis BJ, et al. A tissue biomarker panel predicting systemic progression after PSA recurrence post-definitive prostate cancer therapy. PLoS One. 2008;3(5):e2318.

60. Cooperberg MR, Simko JP, Cowan JE, Reid JE, Djalilvand A, Bhatnagar S, et al. Validation of a cell-cycle progression gene panel to improve risk stratification in a contemporary prostatectomy cohort. J Clin Oncol. 2013:31(11):1428-34.

61. Knezevic D, Goddard AD, Natraj N, Cherbavaz DB, Clark-Langone KM, Snable J, et al. Analytical validation of the Oncotype DX prostate cancer assay - a clinical RT-PCR assay optimized for prostate needle biopsies. BMC Genomics. 2013;14:690

62. Chen Y, Wen D, Huang Z, Huang M, Luo Y, Liu B, et al. 2-(4-Chlorophenyl) -2-oxoethyl 4-benzamidobenzoate derivatives, a novel class of SENP1 inhibitors: Virtual screening, synthesis and biological evaluation. Bioorg Med Chem Lett. 2012;22(22):6867-70

63. Madu IG, Namanja AT, Su Y, Wong S, Li YJ, Chen Y. Identification and characterization of a new chemotype of noncovalent SENP inhibitors. ACS Chem Biol. 2013;8(7):1435-41.

64. Qiao Z, Wang W, Wang L, Wen D, Zhao Y, Wang Q, et al. Design, synthesis, and biological evaluation of benzodiazepine-based SUMO-specific protease 1 inhibitors. Bioorg Med Chem Lett. 2011;21(21):6389-92.

\section{Submit your next manuscript to BioMed Central and take full advantage of:}

- Convenient online submission

- Thorough peer review

- No space constraints or color figure charges

- Immediate publication on acceptance

- Inclusion in PubMed, CAS, Scopus and Google Scholar

- Research which is freely available for redistribution

Submit your manuscript at www.biomedcentral.com/submit 